\title{
FORUM
}

\section{Assoziierung plus gesamteuropäische Aufgabenkonföderation: Plädoyer für eine selbstbewusste EU-Nachbarschaftspolitik}

\author{
Barbara Lippert*
}

Die EU befindet sich in einem Dilemma zwischen der außenpolitisch in mancher Hinsicht gebotenen und vielfach erwarteten Fortführung des Erweiterungsprozesses und den europapolitischen Notwendigkeiten einer Konsolidierung ihrer Grundlagen. ${ }^{1}$ Für die EU gilt es vor diesem Hintergrund, die eigene Glaubwürdigkeit nach innen und außen zu stärken und ihre Handlungsspielräume zu erweitern.

Rhetorische Frontstellungen zwischen Gegnern und Befürwortern einer Fortsetzung der Erweiterungspolitik unter den Mitgliedstaaten führen die EU nur weiter in die Sackgasse. Während die einen die politische, finanzielle und institutionelle Absorptionskraft der EU zum alles entscheidenden, wenngleich nur vage ausbuchstabierten Kriterium erheben ${ }^{2}$, verlassen sich die anderen auf das Einfallstor, das Artikel 49 EUV für europäische Staaten bietet und auf die bürokratischen Automatismen, die ein Beitrittsantrag - siehe jüngst den Mazedoniens - für gewöhnlich auslöst. ${ }^{3}$ Nach außen gibt die EU zunehmend ein Bild der Doppelzüngigkeit und zugleich der Erpressbarkeit ab. Die 25 können sich weder dazu durchringen, kollektiv Ja zur fortgesetzten Erweiterung zu sagen, noch kann die EU wohl auf Dauer ein Nein durchhalten. In dieser angespannten Situation geht es für die EU darum, Gestaltungskraft zurück zu gewinnen. Nach Lage der Dinge und angesichts divergierender Präferenzen unter den Mitgliedstaaten gibt es keinen Königsweg, sondern nur kluge und weniger kluge Kompromisse. In diesem Sinne ist dies ein Plädoyer für eine Intensivierung der Nachbarschaftspolitik. Dazu werden einige Vorschläge zur Diskussion gestellt. ${ }^{4}$

\section{Die Übergangsphase gestalten: eine Assoziierung neuen Typs}

Mehr noch als die Erweiterung ist die Nachbarschaftspolitik der EU Ordnungspolitik zur Strukturierung und Gestaltung des unmittelbaren Nachbarraums. Gegenüber den Ländern des westlichen Balkans hat die EU jüngst in Salzburg deren europäische Perspektive bekräf-

* Dr. Barbara Lippert, Stellvertretende Direktorin des Instituts für Europäische Politik, Berlin; Lehrbeauftragte an der Humboldt Universität zu Berlin.

Die Vorschläge wurden erstmals im März 2006 im Berliner Arbeitskreis Europa der Friedrich-Ebert-Stiftung diskutiert. Die Autorin dankt den AK-Mitgliedern für ihre Kommentare und Anregungen. Eine weitere Ausarbeitung soll im Sommer von der FES publiziert werden. Dieser Forums-Beitrag resultiert auch aus Arbeiten im Rahmen des IEP-Schwerpunktprogramms „Dialog Europa der Otto Wolff-Stiftung“.

1 Vgl. Schlussfolgerungen des Vorsitzes, Europäischer Rat vom 15./16. 12. 2005, Punkt 25.

2 Das Europäische Parlament hat nun die Kommission aufgefordert, bis Ende 2006 einen Bericht vorzulegen, in dem sie die Prinzipien des „Konzepts der Aufnahmefähigkeit“ erläutert. Vgl. den Bericht des Ausschusses für Auswärtige Angelegenheiten ,über das Strategiepapier 2005 der Kommission zur Erweiterung“ vom 3.2.2006, A6-0025/2006 (Bericht Brok), S.4, Punkt 5.

3 Vgl. zu den Positionen in den 25 Mitgliedstaaten Institut für Europäische Politik (Hrsg.): EU-25 Watch, No. 2, January 2006, Berlin, S. 105-147, abrufbar unter: http://www.iep-berlin.de/publik/EU25-Watch/index.htm (letzter Zugriff: 27.03.2006).

4 Vgl. zum Problemkreis insgesamt mit dem Vorschlag der Aufgabenkonföderation Barbara Lippert: Erfolge und Grenzen der technokratischen EU-Erweiterungspolitik, in: Amelie Kutter/Vera Trappmann (Hrsg.): Das Erbe des Beitritts: Mittel- und osteuropäische Gesellschaften nach dem Beitritt zur EU, Baden-Baden 2006 (im Erscheinen). 
tigt und verhandelt bereits mit Kroatien über den Beitritt. ${ }^{5}$ Gleiches gilt für die Türkei. Eine EU von 30 Mitgliedstaaten zeichnet sich so für einen mittleren Zeitraum von zehn bis fünfzehn Jahren ab. Hinter diese zugesicherten Beitrittsperspektiven sollte die EU auch von sich aus nicht zurückfallen. Die Mittelmeerländer sind überwiegend bereits mit der EU assoziiert und multilateral im Barcelona-Prozess verbunden. Unter den östlichen Nachbarn reklamiert Russland eine Sonderstellung und bequemt sich, mit der EU vier Räume der Kooperation zu schaffen. ${ }^{6}$ Dazwischen liegen jene postsowjetischen Länder, die, wie die Ukraine, bereits historisch Grenzräume Europas bilden und die sich noch inmitten langwieriger Transformations- und Orientierungsprozesse befinden. Unterdessen ist aber auch für nennenswerte Teile der Gesellschaften zwischen Minsk und Baku und einige ihrer Regierungen die EU zu einem Fixpunkt der Reformanstrengungen und außenpolitischen Ausrichtung geworden. Sie treten der EU mit großen Erwartungen entgegen und verlangen, dass sie nichts tun solle, was ihre Hoffnung auf EU-Mitgliedschaft, und liegt sie auch in noch so weiter Ferne, ,tötet"

Angesichts dieser Erwartungen muss die EU ihrer Nachbarschaftspolitik endlich Zugkraft und Attraktivität verleihen. Durch spezifische Angebote und Neuerungen kann sie die durch Erweiterungsmüdigkeit und gebremste Integrationsdynamik ${ }^{8}$ geprägte (Übergangs-) Zeit aktiv gestalten. Je länger diese Übergangsphase dauert und je weniger bei einigen Ländern an die ,Perspektive einer Perspektive' zu denken ist, desto dringender wird sich diese Aufgabe stellen. Das gilt vor allem für den postsowjetischen Raum. Zur Schärfung ihrer östlichen Dimension könnte die EU folgende Schritte tun.

Hauptinstrument sind individuell mit den Nachbarn auszuverhandelnde bilaterale Abkommen, die durch Aktionspläne unterfüttert und operationalisiert werden. An die Stelle der 2008 auslaufenden Partnerschafts- und Kooperationsabkommen (zum Beispiel mit der Ukraine und Moldau) sollten Assoziierungsabkommen neuen Typs ${ }^{9}$ treten. Das Angebot zielt prinzipiell auf die Ukraine, Moldau, Belarus, Georgien, Armenien und Aserbaidschan. Das Assoziierungsabkommen neuen Typs (basierend auf Artikel 310 und 300 EGV) wird als Modernisierungs- und Stabilitätspartnerschaft konzipiert. Der Grad der Konvergenz mit dem Unions-Acquis wird individuell nach Ambition und Leistungskraft anvisiert. Diese Assoziierung unterscheidet sich mithin vom Typus der Beitrittsassoziierung wie im Falle der Türkei oder Griechenlands und der bloßen Freihandelsassoziierung wie bei den Mittelmeerländern.

Im folgenden wird nichts grundstürzend Neues vorgeschlagen, sondern traditionelle Elemente mit Angeboten kombiniert, die substantiell über den Status quo hinausgehen und entsprechende Anreize setzen. Für die Modernisierungs- und Stabilitätspartnerschaft sind in den neuen Assoziierungsabkommen maßgeschneidert und im Lichte der spezifischen Ambitionen für das jeweilige Land die in Übersicht 1 genannten Elemente vorzusehen. ${ }^{10}$

5 Vgl. die Gemeinsame Presseerklärung EU/Western Balkans vom 11.03.2006, abrufbar unter: http://www.eu 2006.at/de/News/Press_Releases/March/1103EUWesternBalkansStatement.html (letzter Zugriff: 21.03.2006).

6 Vgl. Hannes Adomeit/Rainer Lindner: Die „Gemeinsamen Räume“ Russlands und der EU. Wunschbild oder Wirklichkeit?, SWP-Studie Nr. 34, 2005.

7 So publikumswirksam der ukrainische Schriftsteller Juri Andruchowytsch in seiner Rede „Europa, meine Neurosen“ bei der Entgegennahme des Leipziger Buchpreises zur Europäischen Verständigung 2006. Auszugsweise abgedruckt in: Süddeutsche Zeitung, 16.03.2006.

8 Vgl. u.a. die Position der SPD-Abgeordneten im Europäischen Parlament zur EU-Erweiterung vom 07.03.2006 (Hänsch/Kuhne), abrufbar unter: http://www.spd-europa.de/index.php?pageId=20\&news_id=511 (letzter Zugriff: 21.03.2006) und Ausschuss für Auswärtige Angelegenheiten, Bericht Brok.

9 Vgl. zum Vorschlag der Nachbarschaftsabkommen als Assoziierungsabkommen neuen Typs Barbara Lippert: Die Union und ihre Nachbarn nach dem Verfassungsvertrag, in: Mathias Jopp/Saskia Matl (Hrsg.): Der Vertrag über eine Verfassung für Europa. Analysen zur Konstitutionalisierung der EU, Baden-Baden 2005, S. 367-379, hier S. 376.

10 Vgl. die Resolution des Europäischen Parlaments zur europäischen Nachbarschaftspolitik vom 19.01.2006, A6-0399/2005, in der einige der hier genannten Elemente im Hinblick auf einen neues Nachbarschaftsabkommen enthalten sind. Allerdings wird weder eine Gesamtkonzeption entwickelt, noch eine klare Einordnung als Assoziierungsabkommen vorgenommen. 


\section{Übersicht 1: Elemente der Assoziierungsabkommen neuen Typs}

- Wirtschaft und Handel

- asymmetrische Handelsliberalisierung, schrittweise einschließlich von Agrargütern und Dienstleistungen

- Option: schrittweise Schaffung einer Freihandelszone mit der EU/dem EWR und schrittweise wirtschaftliche Integration in den EU-Binnenmarkt

- Finanzielle und technische Hilfspakete für wirtschaftliche Transformation (economic governance und good governance)

- Investitionshilfen der EU und der Privatwirtschaft für Infrastrukturprojekte: Verkehr (besonders Schiene), Energie, Telekommunikation

- Demokratieförderung und politischer Dialog

- politische Konditionalitätsklausel

- vertiefter politischer Dialog im Rahmen der Assoziierungsinstitutionen zu den Themen good governance, Menschenrechte, Demokratie

- weitere politikfeldspezifische Themendialoge

- Unterstützung beim Institutionenaufbau, insbesondere im Bereich des Gerichtswesens und der Justiz sowie generell der öffentlichen Verwaltung

- Ausbau von TAIEX und TwinningAktivitäten

- Systematische Einbeziehung von zivilgesellschaftlichen Vereinigungen und der Sozialpartner als Dialogund Kooperationspartner

- Öffnung von EU Programmen, vor allem der Mobilitätsprogramme, und vereinfachtes Visaregime für Studierende und Lernende
- Außen- und sicherheitspolitischer Dialog und Kooperation

- Möglichkeit der Assoziierung mit gemeinsamen Stellungnahmen und Aktionen im Rahmen der GASP

- Teilnahme an gemeinsamen Missionen und Einsätzen

- Politischer Dialog: regelmäßig auf Minister-Ebene, hohe Beamte; darüber hinaus fallweise: Gipfel, informelle Treffen (Typ Gymnich); Teilnahme an GASP-Arbeitsgruppen, Politisches und Sicherheitspolitisches Komitee, gemeinsame Ratssitzungen zu spezifischen Themen

- Themen: regionale Sicherheit, Krisenmanagement, Nichtverbreitungspolitik, Bekämpfung des internationalen Terrorismus, Energiesicherheit

- Innere Sicherheit

- Bekämpfung organisierter Kriminalität

- Grenzsicherheit

- Einwanderungspolitik

- Rückführabkommen

- Visapolitik

- Kooperation mit Behörden und EUAgenturen

- Hilfe

- Der Schwerpunkt sollte auf Maßnahmen gelegt werden

- zur Verbesserung von economic und good governance

- zum Ausbau von Infrastruktur

- zur grenzüberschreitenden Kooperation und Verflechtung (siehe CEFTA)

- zur regionalen Entwicklung in den Ländern. 
Sinn und Zweck der immer enger auszugestaltenden Assoziierung wäre die Modernisierungs- und Stabilitätspartnerschaft, nicht der Beitritt. Die Frage der EU-Mitgliedschaft wird seitens der EU ausgeklammert. Sie stellt demgegenüber eine effektive Verbesserung der Lebensverhältnisse und verlässliche politische Beziehungen ins Zentrum. Maßnahmen und Themen, die innerhalb der fünf vorgeschlagenen Kooperationsfelder (siehe Übersicht 1 oben) ausgewählt und zeitlich sowie inhaltlich ausgestaltet werden, sind auf das Ziel der Modernisierung ausgerichtet. Zentrales Anliegen und Gradmesser ist also die effektive Unterstützung der Transformation in Bezug auf good governance und wirtschaftliche Entwicklung ${ }^{11}$, nicht die Konvergenz mit dem EU-Acquis von der Gemeinsamen Agrarpolitik bis zur Wettbewerbspolitik. Die Komponente „Stabilitätspartnerschaft“ unterstreicht das zunehmende Interesse der EU an der Befriedung und Entspannung regionaler Konflikte in Osteuropa, die teilweise eine Voraussetzung für Demokratisierung und wirtschaftliche Entwicklung in der Region sind. ${ }^{12}$ Die Stabilitätspartnerschaft betont außerdem das Interesse der EU, diese Länder für den Kampf gegen organisierte Kriminalität und die Weiterverbreitung von Massenvernichtungswaffen, für die Sicherung der Energielieferungen und die Abwehr des internationalen Terrorismus, mithin für die Herausforderungen der global governance, auch angesichts aufsteigender Mächte wie China und Indien, zu gewinnen.

Die Frage der institutionellen Ausgestaltung der Assoziierung und der Beteiligung der Nachbarn am EU-Entscheidungssystem ist wichtig, weil sie in der Regel die rote Linie zwischen „,drinnen“ und „draußen“ markiert und für Drittstaaten eine Frage des besonderen Status und Verhältnis zur EU darstellt. Vorgeschlagen wird eine stärkere Einbeziehung der Nachbarschaftspolitik-Staaten in den Meinungsbildungsprozess in der EU (,decision shaping', aber kein ,decision making') durch Rückgriff auf und Überarbeitung der strukturierten Zusammenarbeit. ${ }^{13}$ Dazu zählen in einer maximalen Variante:

- Gipfel (Troika trifft Staats-/Regierungschef)

- Assoziierungsrat auf Minister-Ebene

- Assoziierungsausschuss Ebene hohe Beamte/Kommission und Unterausschüsse

- Parlamentarischer Assoziierungsausschuss

- Zivilgesellschaftliche Foren

- (thematische)Verknüpfung mit der „Aufgabenkonföderation“ (siehe unten)

- Einbeziehung als Beobachter (mit Rederecht und Informationsrechten) in Vorbereitungsund Implementierungsstadien des Legislativprozesses (zum Beispiel nach dem Muster des EWR)

- Back to back meetings bi- und multilateral mit Europäischem Rat und ausgewählten Ministerrat-Konfigurationen (Allgemeine Angelegenheiten und Sektorpolitiken).

Diese Parallelstrukturen sind für die EU aufwändig und für Drittstaaten nur ein Ersatz für volle Mitgliedschaftsrechte. Auch deshalb wurden sie in der Vergangenheit eher obstruiert als engagiert genutzt. Der EU müsste nun aber klarer vor Augen stehen, dass sie den Preis einer intensiven und substantiellen Nutzung derartiger Konsultationsvorkehrungen zahlen muss, wenn sie die Assoziierung attraktiver gestalten und als Alternative zum Beitritt aus-

11 So auch die Argumentation des Generaldirektors der GD Außenbeziehungen der Europäischen Kommission Eneko Landaburu: "From Neighbourhood to Integration Policy: are there concrete alternatives to enlargement?", Brüssel, 23.01.2006, abrufbar unter http://europa.eu.int/comm/world/enp/pdf/060223_el_ceps_en.pdf (letzter Zugriff: 21.03.2006).

12 Auf diesen Zusammenhang verweist Ernst-Otto Czempiel: Demokratisierung von außen. Vorhaben und Folgen, in: Merkur, Nr. 6/2004, S. 467-479, hier S. 472.

13 Zur strukturierten Zusammenarbeit vgl. Barbara Lippert/Peter Becker: Bilanz und Zukunft des Strukturierten Dialogs, in: integration 2/1997, S. 56-71. 
bauen will. Die Nachbarn könnten ihrerseits zur Einsicht gelangen, dass diese Arrangements kein schwacher Ersatz sein müssen. Vor allem dann nicht, wenn man sieht, dass derzeit schon Abstriche am Konzept der Vollmitgliedschaft bei laufenden Beitrittsverhandlungen gemacht und für potenzielle Kandidaten Formen abgestufter Mitgliedschaft kreiert werden sollen. ${ }^{14}$

$\mathrm{Zu}$ vermitteln ist, dass dieser neue Assoziierungstyp erheblich mehr als die Partnerschafts- und Kooperationsabkommen (zum Beispiel wegen der pfeilerübergreifenden Themenbreite, der institutionellen Ausgestaltung und der ,Hilfepakete'), aber weniger beziehungsweise anderes als die Europaabkommen bietet (siehe Beitritt) oder fordert (siehe Konvergenzanforderungen). Die Beziehungen zwischen der EU und den Assoziierten sind symmetrischer angelegt und stehen nicht unter dem Imperativ der einseitigen Acquisübernahme. Sie sind dadurch (beiderseits) selektiver und stärker als interessegeleitete Verhandlungen (mit Geben und Nehmen) konzipiert. Deshalb wäre auch weniger an einen maximalen politisch-wirtschaftlichen Kriterienkatalog wie an den von Kopenhagen für Beitrittskandidaten zu denken, der ja einen beitrittsfixierten Stufenprozess auslöst. Stattdessen würde eine Serie zweiseitiger Vereinbarungen (Aktionspläne, aber auch völkerrechtliche Vereinbarungen und politische Übereinkünfte) und ein an Benchmarks orientiertes Monitoring im Rahmen der Assoziierungsinstitutionen verfolgt. Die Ukraine könnte als Zugpferd und Pilotland für eine solche Assoziierung neuen Typs aufgebaut werden.

\section{Perspektive gesamteuropäische Aufgabenkonföderation}

Darüber hinaus bedarf es aber einer weiteren konzeptionellen Perspektive für die Nachbarstaaten, die möglicherweise auch auf Dauer der EU nicht beitreten werden. Die Assoziierungsabkommen neuen Typs sollten der Baustein für eine umfassende Neuordnung der Beziehungen zwischen der sich ohnehin - siehe Balkan - erweiternden EU und den östlichen Nachbarn sein. Kernidee ist es, eine ,gesamteuropäische Aufgabenkonföderation“ ${ }^{15} \mathrm{zu}$ schaffen, die in der Hauptsache eine funktionale Kooperation unter Einmischung gemeinsamer Entscheidungsverfahren und ,leichter Institutionen' wäre. Es handelt sich einerseits um einen Zweckverband, anderseits ist die Zusammenarbeit langfristig angelegt und institutionalisiert und kann, soweit erforderlich oder gewünscht, ,auch mit gemeinsamer Ausübung von Hoheitsgewalt der Mitglieder einhergehen" $" 16$.

In der Aufgabenkonföderation sollte die EU kollektiv (beziehungsweise entsprechend ihrer Kompetenzordnung) vertreten sein und von den postsowjetischen Nachbarn jene, die mit

14 Vgl. hier z.B. die Beiträge der Bundestagsabgeordneten Andreas Schockenhoff: Aufnahmefähigkeit der EU klären, 18.02.2006, abrufbar unter: http://www.cducsu.de/section_2/subsection_3/id_1153/Meldungen.aspx (letzter Zugriff: 24.03.2006); Mathias Wissmann: Neue Strategien für die Erweiterung der EU - Das Modell der gestuften Mitgliedschaft" - Thesenpapier zur Zukunft der Erweiterung der europäischen Union, 16.3.2006, abrufbar unter: http://www.matthias-wissmann.de/images/content/20060316_handout_wissmann. pdf (letzter Zugriff: 24.03.2006) sowie die SPD-Abgeordneten im Europäischen Parlament, Position zur EUErweiterung, Punkt 2.

15 Begriff und Ausgangsidee in Anlehnung an den Bericht von Hänsch für den Institutionellen Ausschuss des Europäischen Parlaments „Über die Gestaltung und Strategie der Europäischen Union im Hinblick auf ihre Erweiterung und die Schaffung einer gesamteuropäischen Ordnung“ vom 21.05.1992, A3.0189/92 (Bericht Hänsch). Damit wird an dieser Stelle von einer eingehenderen Diskussion des Begriffs Konföderation im staatsrechtlichen Sinne und den jeweiligen Bedeutungsinhalten abgesehen. Vgl. Heinrich Schneider: Optionen der politischen Finalität: Föderation - Konföderation - Verfassung, in: ders., Mathias Jopp und Uwe Schmalz (Hrsg.): Eine neue deutsche Europapolitik?, Bonn 2001, S. 583-666.

16 Institutioneller Ausschuss, Bericht Hänsch, S. 24. Gegen den Begriff Konföderation hatten sich in den Beratungen des Verfassungskonvents allerdings vor allem Vertreter der polnischen Regierung gewandt, vgl. Lippert, Die Union und ihre Nachbarn, S. 372-375. 
der EU assoziiert sind, die eine gute Umsetzungsbilanz aufweisen und die die ambitionierten Kooperationsziele der Modernisierungs- und Stabilitätspartnerschaft erfolgreich verfolgen. Die Einbeziehung Russlands und die Verbindung zur Politik der vier gemeinsamen Räume wäre als weitere wichtige Strukturfrage zu diskutieren.

Fortgeschrittenen Assoziierungsländern bietet die EU an, mit ihr eine gemeinsame, tendenziell gesamteuropäische Aufgabenkonföderation zu bilden. Sie dient dazu, bilaterale Themen übergreifend und multilateral zu behandeln und, nach festzulegenden Verfahren, gemeinsam Entscheidungen zu treffen. Diese Konföderation sollte einen Prozess initiieren, in dessen Verlauf gemeinsame Institutionen (zum Beispiel Konföderationsrat auf Ebene von Ministern, hohen Beamten und der Parlamente) geschaffen und Aufgaben, Verfahren, Regeln und Normen der Kooperation bis hin zur ,Gemeinschaftsbildung' allmählich fest- und fortgeschrieben werden. Ähnlich wie beim KSZE-Prozess stünde das Ergebnis nicht im Vorhinein fest. Vielmehr ginge es darum, diese gesamteuropäische Aufgabenkonföderation auf den Weg zu bringen und ihr dann allmählich eine festere Struktur zu geben. Dies nicht zuletzt vor dem Hintergrund einer noch andauernden Neustrukturierung des internationalen Systems und der internationalen Organisationen. Die Einbindung des Europarats und gegebenenfalls auch die Nutzung seiner Infrastruktur für diese Aufgabenkonföderation lägen nahe. Andere Organisationen wie die NATO und seit langem schon die OSZE sind raumgreifender angelegt als die Konföderation, aber ebenso in einem Wandlungsprozess begriffen.

Im Unterschied zum Stabilitätspakt für den westlichen Balkan würde allerdings innerhalb der Aufgabenkonföderation keine Proliferation von internationalen Organisationen und Staaten außerhalb Europas eingeplant, da dies zu einer schwerfälligen Struktur führt. Vielmehr sollten die räumliche Begrenzung auf die jetzige Liste der sechs postsowjetischen Nachbarschaftsländer ( potenziell plus Russland plus EFTA-Länder) und die zentrale Stellung der EU die politische Attraktivität der Aufgabenkonföderation herausstreichen.

Im Vergleich zum Barcelona-Prozess würden funktionierende bilaterale Assoziierungsbeziehungen das Rückgrat der sie krönenden beziehungsweise fortsetzenden Aufgabenkonföderation bilden und nicht umgekehrt lahme bilaterale Beziehungen multilateral dupliziert. Die hier vorgeschlagenen substantiellen Assoziierungsabkommen eröffnen bereits bei intensiver Nutzung und institutioneller Ausgestaltung die Möglichkeit zur ,Assoziierung mit EG/ EU-Politiken', wie sie heute vor allem im EWR mit EFTA-Staaten oder bilateral mit der Schweiz praktiziert werden und sich - siehe Schengen - nicht auf Handel und Wirtschaft beschränken.

Im Unterschied zum EWR hätte die Aufgabenkonföderation nicht ausschließlich eine wirtschaftliche Dimension. Ja, das im EWR intendierte schwache politische Profil sollte in der Aufgabenkonföderation ins Gegenteil gekehrt und ausdrücklich mit der Aussicht auf eine Gemeinschaftsbildung im Sinne von Karl W. Deutsch lanciert werden. ${ }^{17}$ Andererseits wäre die gesamteuropäische Aufgabenkonföderation aufgrund der Leistungskraft der Nachbarn und der je spezifischen Interessenlage der Beteiligten aber auch weniger ambitioniert in den Konvergenzzielen als der EWR, soweit sie beispielsweise den Binnenmarkt und die Wettbewerbspolitik betreffen. Die Ausweitung der Handlungsfelder (zum Beispiel auf in-

17 Vgl. Karl W. Deutsch u.a.: Political Community and the North Atlantic Area, Princeton 1957, S. 66f. zum Modell der pluralistischen Sicherheitsgemeinschaft. Zu deren Hauptbedingungen zählen: die Kompatibilität von Grundwerten, die Responsivität gegenüber dem Partner sowie die wechselseitige Voraussagbarkeit des Verhaltens. Vgl. zur Übertragung auf die OECD-Welt: Dieter Senghaas: Friedenszonen, in: Karl Kaiser/Hans-Peter Schwarz (Hrsg.): Die neue Weltpolitik, Bonn 1995, S. 337-346. 
nere Sicherheit, GASP/ESVP) und ihre Verschränkung unterstreicht hingegen die politische Dimension der gesamteuropäischen Aufgabenkonföderation.

Im Zentrum der gesamteuropäischen Aufgabenkonföderation stünden drei Körbe/Dimensionen und acht ,Räume':

- die politische und humanitäre Dimension

- Rechtstaatlichkeit, Demokratie und Menschenrechte (aufbauend auf den EuroparatMitgliedschaften)

- Bildung, Kultur und Wissenschaft (zum Beispiel anknüpfend an den Bologna-Prozess)

- die ökonomische Dimension

- ökonomischer Raum (Außenhandel und interne Marktregulierung)

- monetärer und makroökonomischer Raum (Euro und makroökonomische Politik)

- ökonomische Infrastruktur / transeuropäische Netze (Transport, Telekommunikation, Energie, Umwelt)

- die Sicherheitsdimension

- Justiz und Inneres (unter anderem Voraussetzungen für Schritte in Richtung von Visafreiheit und Öffnung von Arbeitsmärkten)

- externe und Sicherheitspolitiken (unter anderem eingefrorene Konflikte)

- Bündelung von regionalen Prozessen.

Im Vergleich zu anderen Vorschlägen ${ }^{18}$ soll nicht primär ein neuer, dritter Status zwischen Mitgliedschaft und Nachbarschaft geschaffen werden. Der Akzent würde vielmehr darauf liegen, dass die EU mit den assoziierten Nachbarn eine Konföderation der Europäer gründet. Damit würde die EU eine intensive Verbindung mit den Nachbarn eingehen. Assoziierung plus Aufgabenkonföderation werden nicht in erster Line als Durchgangsstation zur Mitgliedschaft angeboten, sondern als eine funktionierende Gemeinschaft von eigenem Wert aufgebaut. ${ }^{19}$

Für die EU liegen die Vorteile auf der Hand: Die gesamteuropäische Aufgabenkonföderation wäre als temporäre, möglicherweise auch dauerhafte Alternative zur EU-Mitgliedschaft für Osteuropa und den Südkaukasus bis hin zu Russland zu entwickeln. Die EU könnte so zunächst ihren inneren Zusammenhalt und ihre Handlungsfähigkeit verbessern, ohne durch Notaufnahmen und Automatismen zur endlosen Erweiterung verurteilt zu sein. Ferner könnte sie in dieser Konsolidierungsphase darüber entscheiden, ob sie erstmals primärrechtliche Möglichkeiten der Teilmitgliedschaft von Drittstaaten und damit der teilweisen Mitentscheidung und Repräsentation in den EU-Organen schaffen will. Nach dem hier vorgeschlagenen Modell der Assoziierung plus Aufgabenkonföderation gäbe es für die Nachbarn nur ein Opt-in bei der Vorbereitung und Implementierung, nicht der Entscheidungsfindung in der Union. Die Aufgabenkonföderation will ja gerade diesem Manko durch eine neue Entscheidungsstruktur außerhalb der EU begegnen.

Die Aufgabenkonföderation wäre zudem eine Rückfalloption für Kandidaten, deren Beitrittsverhandlungen nicht erfolgreich verlaufen. Im Sinne konzentrischer Kreise wäre ein

18 Vgl. Bericht über das Strategiepapier (Bericht Brok), Erwägungspunkt 10 über einen „multilateralen Rahmen als Zwischenschritt hin zu einer Vollmitgliedschaft", sowie die Ausführungen auf der Pressekonferenz: Agence Europe, 16.03.2006, S. 5; außerdem Wissmann, Neue Strategien. Interessanterweise plädiert Brok für einen multilateralen Rahmen und Hänsch aktuell dagegen. Vgl. Agence Europe vom 18.03.2006, S. 5.

19 Natürlich ergeben sich hier eine ganze Reihe Fragen, die der weiteren Klärung bedürfen: Wie wird das Verhältnis zwischen Aufgabenkonföderation und EU geregelt, wie das Verhältnis zwischen Aufgabenkonföderation und den Assoziierungen unterschiedlicher Qualität und Intensität mit den Nachbarn praktisch gestaltet? Wer entscheidet über die Aufnahme in die Aufgabenkonföderation? Welche Mittel muss die EU in ihrem Haushalt für die intensivierte Nachbarschaftspolitik bereitstellen? 
Ring ,verantwortungsvoll regierter Staaten“ um die EU (plus EWR) gelegt. Vom äußeren Kreis zum inneren EU-Kreis vorzustoßen ist seitens der EU jedoch nicht auszuschließen, solange sie an Artikel 49 EUV festhält. Eine Alternative wäre, die Antragstellung an eine entsprechende - wohl einstimmig auszusprechende - Einladung seitens der EU zu knüpfen. ${ }^{20}$

\section{Interessenprofil der EU präzisieren}

Parallel zur Effektivierung der Europäischen Nachbarschaftspolitik durch die Assoziierungsabkommen neuen Typs in der Praxis sollten innerhalb der EU konzeptionelle Überlegungen vorangetrieben werden. Erst auf der Grundlage einer präziseren Interessenabklärung kann eine selbstbewusste Nachbarschafts- und Erweiterungspolitik, die wohl immer unterhalb der Erwartungen von Drittländern bleiben, durchgeführt werden. Folgende Punkte sind zu diskutieren:

- die Definition der Interessen der EU an der Aufnahme neuer Mitglieder (welche Länder, welcher Zeitrahmen?) beziehungsweise an der politischen und wirtschaftlichen Verflechtung und Strukturierung der Beziehungen, das heißt über den ,level of ambition' sollte jetzt nachgedacht und reaktive Verhaltensmuster eingedämmt werden

- das Offenhalten/Ausklammern des Endpunktes der Beziehungen und die Vermeidung von Automatismen im Hinblick auf den Beitritt

- mögliche Wechselwirkungen mit einer NATO-Erweiterung in Osteuropa (zum Beispiel um die Ukraine oder Georgien)

- die vor allem auf Stabilität bezogenen und im engeren Sinne GASP/ESVP relevanten Elemente der Nachbarschaftspolitik und die stärker auf Demokratieförderung, wirtschaftliche Entwicklung und good governance ausgerichteten Elemente zu gewichten und hinsichtlich der Anreize (Ressourceneinsatz und andere Angebote), Interessenintensität und Durchsetzungsmöglichkeiten (Sanktionen, Konditionalität) auf EU-Seite realistisch einzuschätzen

- ein kohärenteres und sichtbareres Auftreten durch Sonderbeauftragte mit pfeilerübergreifenden Aufgaben

- der parallele Ausbau der strategischen Partnerschaft der EU mit Russland (Komplementarität, Integrationskonkurrenz, positive/negative spill over Effekte $)^{21}$

- die Interessen der USA in der Region (einschließlich Südkaukasus und Zentralasien), ihre Politik innerhalb der NATO und die machtpolitische Konkurrenz mit Russland

- die Bedeutung der Türkei vor und nach einem möglichen EU-Beitritt für die Europäische Nachbarschaftspolitik und die Beziehungen der EU zu den Nachbarländern

- die wirkungsvolle Kooperation mit anderen Organisationen (OSZE, Europarat, OECD, IWF, NATO) und Netzwerken (Barcelona-Prozess, Schwarzmeerkooperation et cetera).

Diese hier nur in Stichworten angedeuteten Probleme und Aspekte verbindet die Frage nach der Gestalt und der strategischen Rolle der EU in der internationalen Politik der kommenden Dekaden. Denn in gewissem Sinne sind die Nachbarn nur der Tellerrand, über den hinaus die EU blicken muß, um sich und ihre Werte in einer möglicherweise volatilen multipolaren Weltordnung zu behaupten. Insofern könnte gerade die Konturierung einer selbstbe-

20 So Lippert, Die EU und ihre Nachbarn, S. 378.

21 In diesem Zusammenhang wären vor allem die Argumente zu berücksichtigen, die das auch von der EU gegenüber Russland verfolgte „Modell Partnerschaft trotz Gegnerschaft“ als bloß simulierte Partnerschaft kritisieren, die nicht die erhoffte Stabilität in Russland und seinem Umfeld bringen werde. So Lilija Š evcova: Garantiert ohne Garantie. Russland unter Putin, in: Osteuropa 3/2006, S. 3-18, hier 13-16. 
wussten und intensiven Nachbarschaftspolitik ein Testfeld für die Fortentwicklung der Europäischen Sicherheitsstrategie ${ }^{22}$ und ihrer praktischen Schlussfolgerungen für die EU sein.

\section{Deutschlands Rolle}

Deutschland kann innerhalb der EU eine führende Rolle bei der Dynamisierung und Gestaltung der Nachbarschaftspolitik spielen. Ein besonderer Akzent liegt aus deutscher Sicht auf der östlichen Dimension der Nachbarschaftspolitik. Eine Verkopplung der Aufgabenkonföderation mit dem Barcelona-Prozess und einer überarbeiteten EU-Russlandpolitik sollte von Anbeginn mitgedacht werden. Die sich bereits abzeichnende faktische Regionalisierung und Individualisierung der Nachbarschaftspolitik sollte jedoch aus Gründen der innergemeinschaftlichen Interessenbalance nicht dazu führen, die Europäische Nachbarschaftspolitik als Dach für die jeweiligen Aktivitäten gänzlich aufzugeben. Insofern sollte die Bundesrepublik Deutschland mit interessierten Staaten (beispielsweise im Rahmen des Weimarer Dreiecks oder mit den Ostseeanrainern, bilateral mit Polen, Ungarn, Rumänien, sowie Italien) den Dialog auch über die konzeptionellen Gesichtspunkte aktiv suchen. Im Lichte ihrer Fähigkeiten und Interessen sollte sie gegenüber den Zielländern ein kluges Management der Erwartungen betreiben und durch die EU effektiv zu deren Modernisierung und Stabilisierung beitragen. Das auf den Weg zu bringen wäre eine lohnende Aufgabe der deutschen Ratspräsidentschaft.

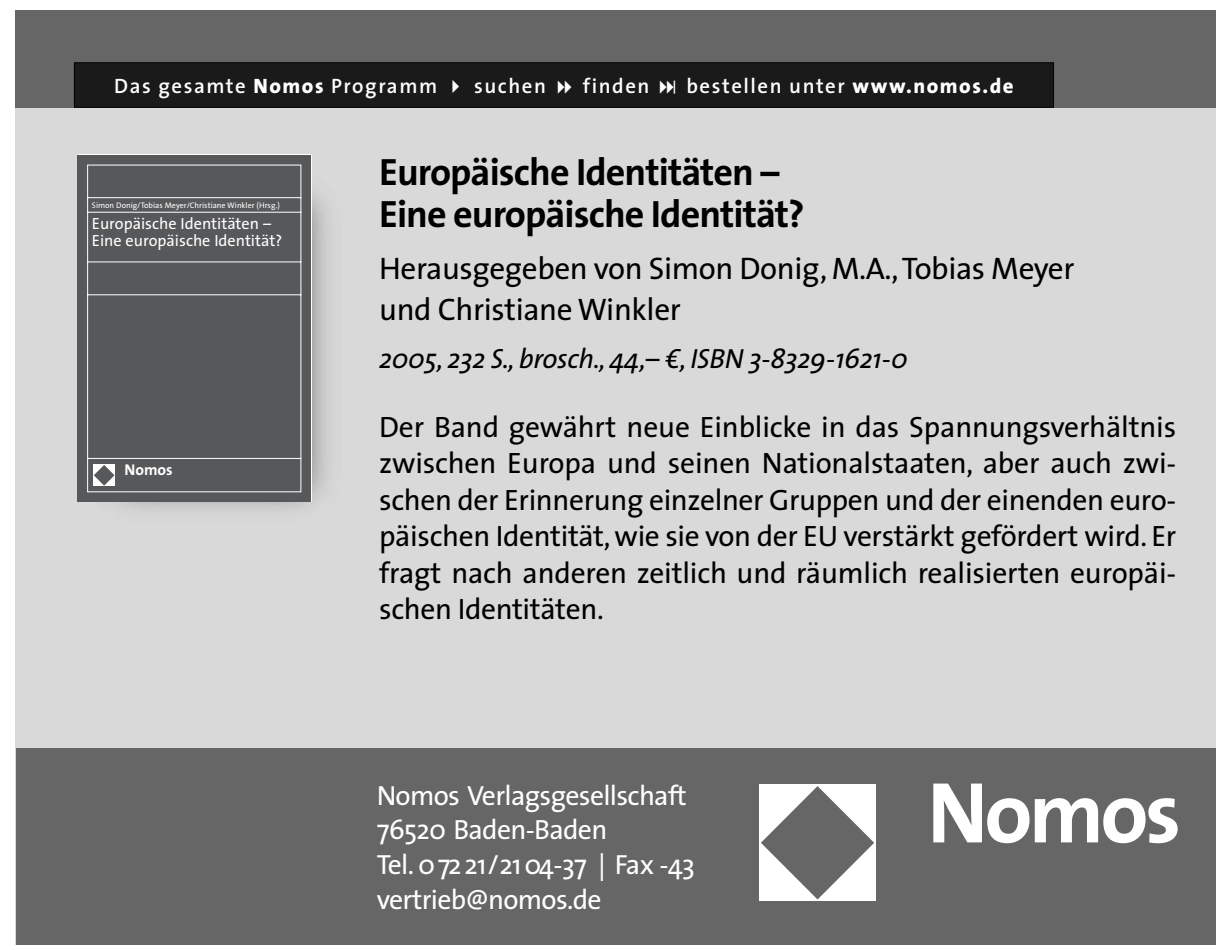

22 Vgl. Europäische Sicherheitsstrategie. Ein sicheres Europa in einer besseren Welt, 12.12.2003, abrufbar unter: http://ue.eu.int/uedocs/cmsUpload/031208ESSIIDE.pdf (letzter Zugriff: 24.03.2006). 